\title{
High permittivity ceramics loaded silicone elastomer composites for flexible electronics applications
}

\author{
L.K. Namitha, M.T. Sebastian
}

\section{Abstract}

The dielectric properties of silicone elastomer composites are important in designing flexible electronic devices. The recent explosive growth in wireless communication, automotive and biomedical applications increases the demand for flexible dielectric materials. However, it is very difficult to identify a homogeneous material which possesses these desired properties. Flexible silicone rubber- ceramic composites based $\mathrm{BaTiO}_{3}(\mathrm{BT})$, $\mathrm{SrTiO} 3(\mathrm{ST})$ and $\mathrm{Ca}_{(1-\mathrm{x})} \mathrm{Nd}_{(2 \times / 3)} \mathrm{TiO}_{3}(\mathrm{CNT})$ ceramic fillers have been prepared. The relative permittivity, thermal conductivity and water absorption increase whereas the coefficient of linear thermal expansion decrease as the volume fraction of filler increases. In the case of dielectric loss; a decreasing trend is shown by SR-ST and SR-CNT composites with filler volume fraction whereas SR-BT composites show a reverse trend since $\mathrm{BT}$ is a lossy material. The composites have $\varepsilon_{\mathrm{r}}$ in the range 3-14 in the microwave frequency range. The composites with high filler loading are suitable candidates for core of flexible dielectric waveguide and embedded capacitor applications and the composites with ST and CNT are suitable for cladding of flexible dielectric waveguide and also for microwave substrate applications

\section{Introduction}

Flexible electronics is an important research area for the next generation consumer electronics and is becoming a part of our day today life [1]. The development of flexible electronic devices continues to advance at great speeds. It is increasingly being used in a number of applications which benefit from their low profile, light weight and favorable dielectric properties. Electronics on flexible substrates are becoming more useful for various devices including sensor arrays/skins, curved circuits and other large area electronics such as flexible displays, capacitors for energy storage, EMI shielding, flexible antenna, electronic paper, low-cost radio frequency identification tags and electronic textiles [2-4]. Compared to flexible electronics built on non-stretchable materials, stretchable materials offer a wide range of advantages such as ability to reduce package size and weight, cost effective installation and dissipation of heat at a higher rate. By replacing the rigid substrate with one that is mechanically flexible will enable a number of unique applications for displays, imagers and large circuits that can be conformably fitted on curved objects and surfaces as needed in biomedical imaging and structural monitoring [5]. In addition to this the flexible materials can cover curved surfaces and movable parts [3]. The stretchable circuits have the ability to withstand large levels of strain without fracture and also no degradation in the electronic properties [4].

The current market analysis estimates that the revenue of flexible electronics can reach 30 billion USD in 2017 and over 300 billion USD in 2028 [6]. The continuous evolution of smaller, lighter and faster electronics necessitated the demand for new materials which can satisfy the requirements of today's electronic world. A flexible dielectric waveguide consists of a flexible core and a flexible cladding. For an ideal flexible waveguide, the core and cladding must be low-loss flexible dielectrics with the relative permittivity of the core being large [7]. Then only the fields of the guided mode would decrease rapidly with distance in the cladding. The requirements for a material to be used as a core of flexible dielectric waveguide are mechanical flexibility, high relative permittivity, low loss tangent, low coefficient of thermal 
expansion (CTE) and high thermal conductivity. It is difficult to obtain a single-phase material with all the above mentioned properties. Recently the research on the development of low loss millimeter wave guiding structures has attained much attention. Recently ceramicpolymer composites have been investigated extensively because of the need for flexible materials with high dielectric permittivity and low cost processing in the rapidly developing electronic industry [8]. However, through the composite approach the permittivity of the polymer can betailored to a greater extent by using high permittivity low loss fillers. Several polymer based dielectric waveguides were developed for flexible electronic applications $[9,10]$. The conventional ceramic polymer composites are flexible to a limited extent but they can't be bend or stretch [11]. Accordingly the importance of the elastomer - ceramic composites, which is receiving wide attention due to its flexibility and stretchability.

The majority of microwave applications are related to high speed microelectronics, radar and communication systems and they need low loss high permittivity materials. High relative permittivity (highk) ferroelectric ceramic-polymer composites have become potential candidate materials for integration into high frequency electronics due to their tailored dielectric, thermal, mechanical properties and ease of processing $[12,13]$. The future of electronics is expected to be soft and rubbery. Recently elastomerceramic composites have been found to be most promising candidates for flexible electronic applications. These composite combines the stretchability and light weight of elastomer with good dielectric and thermal properties of ceramics. Xiang et al. studied the microwave dielectric properties of polyolefin elastomer (POE)-SrTiO3 composites, which can be used as the core of flexible waveguide [14]. Later Thomas et al. and Chameswary et al. reported the high permittivity filler loaded butyl rubber composites for dielectric applications in flexible electronics at microwave frequencies $[15,16]$. Volakis and co-workers studied the ceramic ( $\mathrm{BaTiO}_{3}, \mathrm{Mg}-\mathrm{Ca}-\mathrm{Ti}$ and $\mathrm{Bi}$-Ba-Nd-Titanate) reinforced polydimethyl siloxane (PDMS) composite substrates for conformal microwave applications in the frequency range, $100 \mathrm{MHz}-20 \mathrm{GHz}$ [17]. Recently Salaeh et al. [18] prepared flexible epoxidized natural rubber- $\mathrm{BaTiO}_{3}$ composites and studied the influence of $\mathrm{BaTiO}_{3}$ concentration on cure characteristics, mechanical, dielectric and morphological properties of the composites. High permittivity ceramic materials can be used to make elastomer composites for flexible dielectric waveguide and capacitor applications.

Silicone rubber is a well-known dielectric elastomer which is widely used in various industries due to its favorable dielectric properties, elasticity, biocompatibility, optical transparency, permeability, UV transmission and availability. The Si-O back bone makes them chemically stable even at high temperatures. The molecular structure gives silicones their unique properties, including water-repellency, cold-resistance etc. In the present paper we chose silicone elastomer as the matrix. High relative permittivity materials are used for the manufacture of embedded capacitor as well as dielectric wave guide applications. In order to develop flexible composites with low loss and high relative permittivity the ceramics used should have permittivity much higher than that of rubber matrix. Ceramics such as $\mathrm{BaTiO}_{3}(\mathrm{BT}), \mathrm{SrTiO}_{3}(\mathrm{ST})$ and $\mathrm{Ca}_{(1-x)} \mathrm{Nd}_{(2 \times / 3)} \mathrm{TiO}_{3}$ (CNT) having high permittivity are chosen for the present study. $\mathrm{BaTiO}_{3}$ and $\mathrm{SrTiO}_{3}$ are well studied systems having a perovskite structure. The perovskite titanates like $\mathrm{CaTiO}_{3}$ and $\mathrm{BaTiO}_{3}$ have high relative permittivity but rather high dielectric loss. The perovskite titanates $\mathrm{Ca}_{(1-x)} \mathrm{Nd}_{(2 x / 3)} \mathrm{TiO}_{3}(\mathrm{x}=0.39)$ was used as the low loss high permittivity material. In this paper we report the detailed investigation of silicone elastomer-high permittivity ceramic fillers like $\mathrm{BaTiO}_{3}, \mathrm{SrTiO}_{3}$ and $\mathrm{Ca}(1-\mathrm{x}) \mathrm{Nd}_{(2 \times / 3)} \mathrm{TiO}_{3}$ filled composites to understand their dielectric, thermal and mechanical performance for flexible electronic applications.

\section{Experimental}

In the present work dimethyl end blocked silicone elastomer is used as the matrix and high permittivity fillers such as $\mathrm{BaTiO}_{3}, \mathrm{SrTiO}_{3}$ and $\mathrm{Ca}_{(1-x)} \mathrm{Nd}_{(2 \times / 3)} \mathrm{TiO}_{3}$ used for the composite preparation. The silicone rubber used for the present investigation was methyl end blocked silicone rubber, molecular weight =4, 27,000 $\mathrm{g} / \mathrm{mol}$, provided by Jyothi rubbers, Thrissur, India. The physical and electrical properties of silicone rubber 
are given in Table 1. Commercial grade dicumyl peroxide (DCP) was used as a curing agent. In rubber compounding, it is conventional to use parts per hundred of rubber (phr) to quantify the ingredients used for vulcanization. Ceramic fillers used were prepared by the solid state ceramic route. The Stoichiometric proportion of starting materials were weighed and mixed by wet ball milling using yttria-stabilized zirconia balls and dried. The powder thus obtained were then calcined at appropriate temperatures and ground well. Then it is used for composite preparation with silicone rubber. The raw materials used for the synthesis of $\mathrm{BaTiO}_{3}$ and $\mathrm{SrTiO}_{3}$ are $\mathrm{BaCO}_{3}, \mathrm{SrCO}_{3}$ and $\mathrm{TiO}_{2}$ (99.9+\%, Sigma-Aldrich). The calcination temperature for $\mathrm{BT}$ is $1100{ }^{\circ} \mathrm{C}$ for $4 \mathrm{~h}$ and for ST it is $1200^{\circ} \mathrm{C}$ for $4 \mathrm{~h}$. $\mathrm{Ca}_{(1-x)} \mathrm{Nd}_{(2 \times / 3)} \mathrm{TiO}_{3}$ (CNT) were synthesized using $\mathrm{CaCO}_{3}, \mathrm{Nd}_{2} \mathrm{O}_{3}$ and $\mathrm{TiO}_{2}$ (99.91\%, Aldrich Chemical Company Inc., Milwaukee, WI) of high purity. The CNT ceramic powder was calcined at $1100{ }^{\circ} \mathrm{C}$ for $3 \mathrm{~h}$. Barium titanate $\left(\mathrm{BaTiO}_{3}\right)$ is the first ferroelectric ceramic and a good candidate for a variety of applications due to its excellent dielectric, ferroelectric and piezoelectric properties. It has a perovskite type structure with high relative permittivity $(\approx 1000-2000)$, loss tangent $\left(\approx 10^{-2}\right)$ and high breakdown strength [19]. $\mathrm{BaTiO}_{3}$ is widely studied for its numerous scientific and industrial applications, such as in dielectric capacitors, transducers and tunable phase shifters [20]. Strontium titanate $\left(\mathrm{SrTiO}_{3}\right)$ is a wellknown ceramic; which exists as a centrosymmetric paraelectric material with a perovskite structure at room temperature. It has very large value of $\varepsilon_{\mathrm{r}}(=290)$ and low value $\left(\sim 10^{-3}\right)$ of $\tan \delta$ [21]. $\mathrm{SrTiO}_{3}$ has mixed ionic-covalent bonding properties. This nature of chemical bonding leads to a unique structure, which make it a model electronic material. Ratheesh and coworkers studied the dielectric and mechanical properties of $\mathrm{SrTiO}_{3}$ based PTFE and PEEK composites and found that these composites are suitable for microwave substrate applications $[22,23] . \mathrm{CaTiO}_{3}$ is a perovskite titanate having high relative permittivity of $\sim 170$. However, the high dielectric loss and the large $\tau \varepsilon$ value ( $-400 \mathrm{ppm} /{ }^{\circ} \mathrm{C}$ limits its use in many practical applications [24]. In order to tune the dielectric loss and $\mathrm{tf}$ (temperature stability), the perovskite titanates were substituted with various elements. A significant modification in the properties of this material can be made through rare earth substitution, which has attracted immense attention. Yoshida et al. made substitution of $\mathrm{Ca}$ by trivalent $\mathrm{Nd}$ in $\mathrm{CaTiO}_{3}$ in the range of $0 \leq x \leq 1$ [25]. The loss tangent value decreased with increasing $x$ value. After reaching a minimum value at $x=0.39$, it increased with a further increase in $x$ value. In the present study we made quaternary compound $\mathrm{Ca}_{(1-x)} \mathrm{Nd}_{(2 \times / 3)} \mathrm{TiO}_{3}$ where $\mathrm{x}=0.39$ due to its useful dielectric properties. The $\varepsilon_{\mathrm{r}}$ and $\tau \varepsilon$ values are 98 and $-130 \mathrm{ppm} /{ }^{\circ} \mathrm{C}$ respectively at $\mathrm{x}=0.39$. Even though, the microwave dielectric properties of $\mathrm{Ca}_{(1-x)} \mathrm{Nd}_{(2 x / 3)} \mathrm{TiO}_{3}$ are studied the $\mathrm{Ca}_{(1-x)} \mathrm{Nd}_{(2 \times / 3)} \mathrm{TiO}_{3}$ filled polymer or elastomer composites are investigated for the first time.

The physical and electrical properties of ceramic fillers are given in Table 1. The compounding of silicone rubber ceramic composites was done by sigma blend method using a kneading machine. The process of sequentially adding the ingredients into the raw rubber is termed 'compounding'. The mixing was done in a kneading machine (Plastomek, Quilandy) at room temperature. The silicone rubber was first masticated through the counter rotating sigma blades to make them soft and more processable. Dicumyl peroxide (DCP) was used as vulcanizing agent. Finally appropriate amount of ceramic fillers were added. The mixing was done for about $45 \mathrm{~min}$ with the rotation speed of $25 \mathrm{rpm}$ to get uniform composites. The uniformly mixed composites were hot pressed at $200{ }^{\circ} \mathrm{C}$ for 90 min under a pressure of $2 \mathrm{MPa}$ with appropriate dies. The optimization of temperature and duration were done by studying the cross linking behavior. After hot pressing, the composites with desired shapes were used for characterization.

The powder XRD spectra were recorded using CuKa radiation (PANalyticalX'PertPRO Diffractometer, Almelo, Netherlands). The microstructure of the silicone rubber- ceramic composites was recorded using scanning electron microscope of JEOL JSM $-5600 \mathrm{LV}$, Tokyo, Japan. The composites were quite flexible so they were dipped into the liquid $\mathrm{N}_{2}$ and fractured. The fractured surfaces thus obtained were used for SEM analysis. The stress-strain properties of thecomposites were measured using a Universal Testing Machine (Hounsfield, H5K-S UTM, Redhill, U.K.) with a rate of grip separation of $500 \mathrm{~mm} / \mathrm{min}$ following the American 
standard ASTM D412. Dumbbell shaped samples of width $4 \mathrm{~mm}$ and thickness in the range of 1.5-2 $\mathrm{mm}$ were used for the tensile measurements. The dielectric properties up to $3 \mathrm{MHz}$ are measured by the parallel plate capacitor method with the help of an LCR meter (HIOKI 3532-50 LCR Hi TESTER, Japan) using cylindrical specimens of $11 \mathrm{~mm}$ diameter and 1-2 mm thickness. A vector network analyzer (Agilent Technologies, E5071C, ENA Series Network Analyzer) was used for the microwave measurements with the help of Split Post Dielectric Resonator and samples in the form of thin sheet. The SPDR's operating at 5.155 $\mathrm{GHz}$ and $15.155 \mathrm{GHz}$ were used for the present study. Bending measurements of the composites were carried out by bending the samples manually in such a way that every part of the sample had undergone $180^{\circ}$ bending. The bending cycle was repeated for 125 times and the microwave dielectric properties were measured by SPDR operating at $5.155 \mathrm{GHz}$ after every 25 cycles. The thermal expansion was measured using a Dilatometer (DIL 402 PC, NETZSCH, Selb, Germany). The thermal conductivity was measured by thermal properties analyzer (Flash Line ${ }^{\mathrm{TM}}$ 2000, Anter Corporation, Pittsburgh, USA). Graphite coated samples of diameter $12.57 \mathrm{~mm}$ and thickness $\approx 1 \mathrm{~mm}$ was used for thermal conductivity measurements. The water absorption characteristic of the composites was measured by following the ASTM D 570-98 procedure. The dimensions of the samples used for the water absorption study was $50 \mathrm{~mm} \times 50 \mathrm{~mm} \times 2 \mathrm{~mm}$. The density of the composites was measured using Archimedes method.

\section{Results and discussion}

Silicone rubber-ceramic composites with high permittivity fillers like $\mathrm{BaTiO}_{3}, \mathrm{SrTiO}_{3}$ and $\mathrm{Ca}(1-x) \mathrm{Nd}_{(2 \times / 3)} \mathrm{TiO}_{3}$ loaded composites were prepared as per the recipe given in Table 2. Maximum filler loading of about 0.28 $\mathrm{V}_{\mathrm{f}}$ can be attained for SR-BT composites whereas $0.3 \mathrm{~V}_{\mathrm{f}}$ for SR-ST composites since the density of $\mathrm{BaTiO}_{3}$ is higher than that of $\mathrm{SrTiO}_{3}$. In the case of SR-CNT composites we can mix up to $0.43 \mathrm{~V}_{\mathrm{f}}$ since $\mathrm{Ca}_{(1-x)} \mathrm{Nd}_{(2 \times / 3)} \mathrm{TiO}_{3}$ has a relatively low density. Larger amount of filler leads to inhomogeneous mixing, hardening and difficulty in mixing and also degradation of the mechanical properties [26]. Moreover, the processing step is more difficult when high filler amounts are incorporated. The optimum filler loading also depends on the particle size, distribution and morphology of the filler. Fig. 1 shows the XRD patterns of BT, ST and CNT and the diffraction peaks are in agreement with standard JCPDS patterns 83-1880 for $\mathrm{BaTiO}_{3}, 35-0734$ for $\mathrm{SrTiO}_{3}$ and 88-0082 for $\mathrm{Ca}_{(1-x)} \mathrm{Nd}_{(2 \times / 3)} \mathrm{TiO}_{3}$. No additional peaks are observed in the powder $\mathrm{X}$-ray diffraction patterns, which indicate the phase purity of the powders of $\mathrm{BaTiO}_{3}, \mathrm{SrTiO}_{3}$ and $\mathrm{Ca}_{(1-x)} \mathrm{Nd}_{(2 \times / 3)} \mathrm{TiO}_{3}$. The formulation recipes of high permittivity ceramic reinforced silicone rubber composites and sample designation and corresponding ceramic volume fraction $\left(V_{f}\right)$ are given in Table 2 .

Distribution of filler in the elastomer matrix and the morphology of the composite are studied using SEM. Fig. 2( $\left.A_{1}\right),\left(B_{1}\right)$ and $\left(C_{1}\right)$ shows the SEM micrographs of $\mathrm{BaTiO}_{3}, \mathrm{SrTiO}_{3}$ and $\mathrm{Ca}(1-x) \mathrm{Nd}_{(2 \times / 3)} \mathrm{TiO}_{3}$ ceramic. Fig. $2\left(A_{2}\right),\left(B_{2}\right)$ and $\left(C_{2}\right)$ shows the fractured surface of lower volume fraction composites whereas $\left(A_{3}\right),\left(B_{3}\right)$ and $\left(C_{3}\right)$ shows the fractogram of higher volume fraction composites respectively. The ceramic fillers are observed to be uniformly distributed throughout the elastomer matrix. In polymer-ceramic composites, as the filler loading increases the tendency for agglomeration will also increase due to the decrease in amount of polymer matrix which keeps the filler particles separated from each other and is evident from Fig. 2 . The mechanical flexibility is a prime requirement for a material to be used for flexible applications. The stress strain curves of pure elastomer and the composites are shown in Fig. 3. It is clear that the stress for the same elongation is larger for ceramic filled composite as compared to unloaded elastomer matrix. As the highly brittle ceramic volume increases inside the matrix, the percentage of elongation decreases. Increased filler loading leads to increase in the stiffness of the composite and hence more stress is required for deformation. Filler parameters like size, shape and aspect ratio have a strong impact on mechanical response of the composites. 
Dielectric properties of 0-3 composites as a function of the volume fraction of the ceramic were studied by many authors. Fig. 4 shows the variation of relative permittivity and the dielectric loss of the composites with varying filler fraction at the radio frequency $(1 \mathrm{MHz})$ as well as at microwave frequency $(5 \mathrm{GHz}$ and 15 $\mathrm{GHz}$ ) regions. The dielectric properties of a 0-3 composite using a dielectric as the filler are dependent on the dielectric properties of both the matrix and filler $[11,27]$. Even though fillers have a very large permittivity than that of matrix, the composite cannot attain relative permittivity more than about 15 , since the relative permittivity depends on the rubber matrix.

The dielectric properties of the rubber composites depend on the volume fraction, size, connectivity between the filler particles and shape of the ceramic fillers and also preparation methods [28]. The reason for the relatively low permittivity of the rubber ceramic composites may be due to the depolarization of the filler while incorporating in the rubber matrix since rubber matrix obscures the polarizability of the ceramic filler [29] and the poor connectivity between the ceramic filler particles [28]. Even at maximum filler loading the relative permittivity of elastomer-ceramic composite cannot increase to a very high value [30]. This is because the 0-3 type composite follows an exponential relationship between the volume fraction of the filler and relative permittivity of composite. Logarithm of the relative permittivity of polymer-dielectric ceramic composites ( $\varepsilon r$ composite) is linearly proportional to the volume fraction of the filler (Vf) with the slope dependent on the dielectric properties of both components [13].

The relative permittivity of polymer-ferroelectric ceramic composite cannot exceed 100 even at maximum filler loading [30]. The effective relative permittivity of polymer -filler composite material is dependent not only on the relative permittivity of the polymer and the filler, size and shape of the filler and the volume fraction of the filler, but also on the relative permittivity of the interphase region and volume of the interphase region. As the filler content increases the interfacial area increases and influence the dielectric properties significantly. The variation of relative permittivity and the dielectric loss of the composites with varying filler fraction at the radio frequency region $(1 \mathrm{MHz})$ are shown in Fig. $4(\mathrm{a})$. It is evident from the figure that relative permittivity of all the composites increases with filler fraction. The value of $\varepsilon_{\mathrm{r}}$ increases with filler loading because $\varepsilon_{\mathrm{r}}$ of fillers is relatively higher than that of the matrix. The increased connectivity among the filler particles and between the polymer and filler can also enhance the relative permittivity. In the case of SR-ST and SR-CNT composites the dielectric loss decreases with filler loading which is due to the low dielectric loss of the filler particles. In the case of SRBT composites the dielectric loss increases with filler content. This is due to the high dielectric loss of the BT than that of silicone rubber matrix.

Fig. 4(b) and (c) shows the variations in microwave dielectric properties of SR-BT, SR-ST and SR-CNT composites with filler loading. The trend in dielectric properties is similar to that of the radio frequency region. The relative permittivity increases with filler volume fraction since the fillers have higher $\varepsilon_{\mathrm{r}}$. At a lower concentration, the rubber matrix isolates filler particles from each other and their dielectric properties will not play a dominant role. Moreover, as the ceramic content increases, the particles will get into contact with each other leading to the formation of continuous networks. Consequently dipole-dipole interaction increases and results in increased values of $\varepsilon_{r}$ [31]. The variations in complex permittivity at microwave range are mainly caused by dipolar relaxation. The effective dielectric loss of SRST and SR-CNT composites decreases with filler loading because here the major factor deciding the dielectric loss is the low $\tan \delta$ values of the fillers. Where as in the case of SRBT composites; a reverse trend is observed, dielectric loss increases with filler loading. The reason for this effect is the higher $\tan \delta$ value of the filler. Moreover, the presence of porosity, the inhomogeneous dispersion of the filler particles moisture and the 
interface between the rubber matrix and ceramics can lead to additional loss. The dielectric properties at 5 $\mathrm{GHz}$ and $15 \mathrm{GHz}$ do not show appreciable differences since there is hardly change in polarization mechanisms.

One advantage of the polymer-ceramic composites is that the relative permittivity can be varied by changing the volume fraction of the filler. Hence the prediction of effective dielectric behavior of the composite is important as far as its application in electronic packaging is concerned. Several theoretical models have been developed to estimate the relative permittivity of polymer-ceramic composite structures. The dielectric properties of the composites are influenced not only by the relative permittivity of the components but also by other factors such as the morphology, dispersion and the interaction between the two phases. The theoretical modeling enables the material scientists to arrive at novel composite systems without much experimental iterations. The experimental value of relative permittivity of composites are compared with the theoretical values predicted by several equations like MaxwellGarnet, Jayasundere -Smith, Lichtenecker and effective medium theory (EMT) model using the following equations [29,32-34].

\section{Maxwell-Garnett equation}

$$
\frac{\varepsilon_{e f f}-\varepsilon_{m}}{\varepsilon_{e f f}+2 \varepsilon_{e m}}=v_{f} \frac{\varepsilon_{f}-\varepsilon_{m}}{\varepsilon_{f}+2 \varepsilon_{e m}}
$$

where $\varepsilon_{\text {eff }}, \varepsilon_{f}, \varepsilon_{m}$ are the relative permittivity of the composites, filler and matrix respectively and $v_{f}$ is the volume fraction of the filler.

The Maxwell-Garnett mixing rule was initially used to calculate the effective permittivity of a system where metal particles are encapsulated in an insulating matrix. This mixing rule was modified for polymer-ceramic composites incorporating homogeneous distribution of spherical ceramic particles and the excitation of dipolar character is considered [32].

\section{Jayasundere-Smith equation}

$$
\varepsilon_{e f f}=\frac{\varepsilon_{m}\left(1-v_{f}\right)+\varepsilon_{f} v_{f}\left[\frac{3 \varepsilon_{m}}{\varepsilon_{f}+2 \varepsilon_{m}}\right]\left[1+\left(\frac{3 v_{f}\left(\varepsilon_{f}-\varepsilon_{m}\right)}{\varepsilon_{f}+2 \varepsilon_{m}}\right)\right]}{1-v_{f}+v_{f}\left[\frac{3 e_{m}}{e_{f}+2 e_{m}}\right]\left[1+\left(\frac{3 v_{f}\left(e_{f}-e_{m}\right)}{e_{f}-2 e_{m}}\right)\right]}
$$

Jayasundere-Smith equation is a modification of well-known Kerner equation by including the interactions between neighboring spheres. This equation considers composite as a bi-phase system of dielectric spheres $\left(\varepsilon_{f}\right)$ dispersed in a continuous medium $\left(\varepsilon_{m}\right)$ and is valid only when $\left.\varepsilon_{f}\right) \varepsilon_{m}[29]$.

\section{Lichtenecker equation}

$$
\ln e_{e f f}=\left(1-v_{f}\right) \ln e_{m}+v_{f} \ln e_{f}
$$

The most widely used relation for the prediction of $\varepsilon_{\mathrm{r}}$ is Lichtenecker's logarithmic law of mixing. It considers the composite system as randomly oriented spheroids that are uniformly distributed in a continuous matrix [33].

\section{Effective Medium Theory (EMT)}

$$
e_{e f f}=e_{m}\left[1+\frac{v_{f}\left(e_{f}-e_{m}\right)}{e_{m}+n\left(1-v_{f}\right)\left(e_{f}-e_{m}\right)}\right]
$$


where $\mathrm{n}$ is empirically determined parameter in the EMT model.

In EMT model, composites are treated as an effective medium whose relative permittivity is obtained by averaging over the relative permittivity of the constituents. The basic concept of EMT model is that when a random unit cell (RUC) is embedded in an effective medium it cannot be detected in the electromagnetic experiment. A random unit cell is defined as a core of ceramic surrounded by a concentric shell of the polymer [34]. A correction factor ' $n$ ' is used to compensate for the shape of the fillers and is called morphology factor which is related to ceramic particle and can be obtained empirically. A small value of ' $n$ ' indicates a near spherical shape for the filler, while a high value of ' $n$ ' shows a largely non-spherically shaped particle.

The theoretical models follow certain assumptions which are related to dielectric properties of individual components, the connectivity of polymer-ceramic composites, size and shape of the filler and morphology. Fig. 5 shows the comparison between experimental and theoretical values of relative permittivity at $5 \mathrm{GHz}$ for SR-BT, SR-ST and SR-CNT composites. It can be seen that the theoretical values give the same trend as that of experimental curve. Maxwell Garnet relations predict lower $\varepsilon_{r}$ compared to the experimental data. Reduction in depolarization of filler and increase in relative permittivity, which are not accounted in this formula, hence shows the deviation. For SR-BT composites the Lichtenecker and EMT models are matching well with experimental results [34]. Jayasundere-Smith and Lichtenecker models are valid for low volume fraction of filler loading, the entrapped air and imperfect dispersion of filler in the composites may be the reason $[29,33]$. Generally, the theoretical predictions are valid only for low volume fractions of filler loading $[35,36]$. This is due the imperfect dispersion of ceramic particle in polymer matrix. The EMT model is found to match with experimental values of SR-ST and SR-CNT composites. The morphology factor ' $n$ ' for EMT model is found to be $0.13,0.103$ and 0.205 SR-BT, SR-ST and SR-CNT composites respectively. EMT model fits well with the experimental results. This includes morphology factor ' $n$ ' which is obtained from empirical calculations. Shape of the filler is compensated by the correction factor, $\mathrm{n}$ in the polymer-ceramic composite. In EMT model, composites are treated as an effective medium whose relative permittivity is the average of the relative permittivity of the constituents. The effective permittivity of a composite depends on the various factors such as relative permittivity of individual components in the system, porosity, their volume fractions, size, shape, interphase polarizability and interphase volume fractions [34]. All these parameters cannot be accounted in a single equation. So some models show slight deviations.

Fig. 6 shows the variation of microwave dielectric properties of silicone rubber composites after repeated bending of samples by an angle of $180^{\circ}$. From the figure it is clear that the relative permittivity of the composites is almost independent of bending. The dielectric loss of composites shows marginal variation with bending which is clear from the figure. The prominent deviation of dielectric loss at higher filler loading may be due to the particle agglomeration at higher filler content. Similar behavior in PFCB (perfluorocyclobutene (poly 1,1,1-triphenyl ethane per-fluorocyclobutyl ether)- $\mathrm{BaTiO}_{3}$ composites was observed by Vrejoiu et al. [37]. The bending of the composites does not considerably affect the microwave dielectric properties and hence, they are suitable for flexible electronic applications.

The relative permittivity of polymer composites should be stable within the operational temperature range of electronic devices for practical applications. From the figure it is clear that all the silicone rubber composites are almost stable within the measured temperature range. The temperature dependence of $\varepsilon_{r}$ at $5 \mathrm{GHz}$ of SR-BT, SR-ST and SR-CNT composite is depicted in Fig. 7. It can be observed that the relative 
permittivity of the composites with lower filler loading is almost constant throughout the measured temperature range. As the filler loading increases there is a decrease in relative permittivity with temperature. This may be due to the disturbed polarizability of dipoles with increase in temperature. The difference in CTE of rubber and filler may be also responsible for the decrease in relative permittivity $[38,39]$. The incipient ferroelectric nature of both BT and ST ceramics can also affect the temperature dependence of relative permittivity of SR-BT and SR-ST composites. Incipient ferroelectrics are characterized by increasing permittivity on cooling due to the softening of the lowest frequency polar optical phonon [40].

The variation in coefficient of thermal expansion (CTE) is an important factor for electronic packaging applications. The variation in CTE of ceramic filled silicone rubber composites are shown in the Fig. 8 . The CTE of pure rubber matrix is $\left(266 \mathrm{ppm} /{ }^{\circ} \mathrm{C}\right)$ very much reduced by the addition of ceramic fillers $\mathrm{BT}$, ST and CNT which are having a very low CTE of $5.4,9.4$ and $10.3 \mathrm{ppm} /{ }^{\circ} \mathrm{C}$ respectively. It is clear from the figure that the thermal expansion of all composites are lowered with the addition of ceramic particles. When a composite is heated, the polymer matrix will expand more than that of ceramic fillers. The expansion of matrix will be reduced if the interfaces are capable of transmitting stress. The polymer chains get arrested in the presence of ceramic and unable to expand with temperature. Hence the CTE of a composite is reduced with increase in filler content and composite with strong interface exhibits an additional reduction of CTE [41].

The silicone elastomer possesses a low value of thermal conductivity of about $0.2 \mathrm{Wm}^{-1} \mathrm{~K}^{-1}$. The thermal conductivity of the fillers are much higher than the matrix and obviously there is an expected trend of increase in the thermal conductivity value with the filler addition. The interfacial physical contact between polymer and filler is very critical for a polymer-ceramic composite [42]. The influence of the volume fraction of filler on the thermal conductivity of SR-BT, SR-ST and SR-CNT composites is shown in Fig. 9. It is observed that the thermal conductivity gradually increases with increasing filler content. At low volume fractions the filler particles will disperse randomly in the rubber matrix and a little increase of thermal conductivity. For higher filler content, the particles begin to touch each other and form conductive channels in the direction of heat flow causing an exponential rise in the thermal conductivity. Ceramic fillers seem to improve the thermal conductivity of polymers as they act as conducting channels with lower thermal resistance than the matrix.

The effect of ceramic loading on specific heat capacity and thermal diffusivity of SR-BT, SR-ST and SR-CNT composites are also depicted in Fig. 9. The specific heat capacity decreases whereas thermal diffusivity increases with filler volume fraction. This is due to the effect of ceramic fillers. They have low $C_{p}$ value and high thermal diffusivity compared to the rubber matrix. The result was in agreement with the earlier reports [43].

The dielectric properties are very sensitive to humid environment. Moreover, a high value of water absorption will lead to considerable degradation of mechanical as well as dielectric properties. The variation of water absorption of SR-BT, SR-ST and SR-CNT composites as a function of filler loading is shown in Fig. 10. It is clear from the figure that the water absorption of the composites increases with filler loading. The increase of moisture absorption may be due to the increase in porosity with filler loading and the hydrophilic nature of the ceramic particles. It has been reported that the materials with moisture absorption up to about $0.1 \%$ can be used for electronic packaging applications [44]. In the current study almost all composites exhibit moisture absorption within this limit. 


\section{Conclusions}

Silicone elastomer reinforced with high permittivity ceramic fillers like $\mathrm{BaTiO}_{3}, \mathrm{SrTiO}_{3}$ and $\mathrm{Ca}(1-\mathrm{x}) \mathrm{Nd}_{(2 x / 3)} \mathrm{TiO}_{3}$ and composites were prepared and their dielectric, thermal and mechanical properties are studied as a function of filler volume fractions. The dielectric properties measured at radio frequency and microwave frequencies are found to improve with increase in filler content. The mechanical flexibility of the composites is revealed by the stress-strain curves. The thermal properties also improved with filler loading. SR-BT composite has $\varepsilon_{\mathrm{r}}=13.38$ and $\tan \delta=0.0435$ (at $5 \mathrm{GHz}$ ), CTE $=188 \mathrm{ppm} /{ }^{\circ} \mathrm{C}, \mathrm{TC}=0.4 \mathrm{Wm}{ }^{-1} \mathrm{~K}^{-1}$ and water absorption $=0.116$ vol\% for $0.272 \mathrm{~V}_{\mathrm{f}}$ of BT loading. For $0.305 \mathrm{~V}_{\mathrm{f}}$ of ST content, the SR-ST composites have $\varepsilon_{\mathrm{r}}$ $=14.21$ and $\tan \delta=0.0116$ (at $5 \mathrm{GHz}$ ), $\mathrm{CTE}=142 \mathrm{ppm} /{ }^{\circ} \mathrm{C}$ and TC=1.02 Wm $\mathrm{Wm}^{-1} \mathrm{~K}^{-1}$ and water absorption of 0.089 vol\% and SR-CNT composites achieved $\varepsilon_{\mathrm{r}}$ of 11.90 and $\tan \delta$ of 0.0115 (at $5 \mathrm{GHz}$ ), CTE and TC of $156 \mathrm{ppm} /{ }^{\circ} \mathrm{C}$ and $0.58 \mathrm{Wm}^{-1} \mathrm{~K}^{-1}$ respectively and water absorption of 0.072 vol\% for $0.43 \mathrm{~V}_{\mathrm{f}}$ of CNT. The composites with highest filler loading are suitable candidates for core of flexible dielectric waveguide and embedded capacitor applications. The measured properties indicate that all the other compositions of SR-BT, SR-ST and SR-CNT composites can be used for cladding of flexible dielectric waveguide and also for microwave substrates applications.

\section{References}

[1] P. Agnihotri, M. Jain, R. Bajpai, Flexible Electronics: revolutionizing the Electronics World 7109 (2013) 49-51.

[2] J. Ryu, K.Y. Kim, J.J. Choi, B.D. Hahn, W.H. Yoon, B.K. Lee, Flexible dielectric Bi1.5Zn 1.0Nb 1.507 thin films on a Cu-polyimide foil, J. Am. Ceram. Soc. 92 (2009) 524-527.

[3] A.C. Siegel, S.T. Phillips, M.D. Dickey, N. Lu, Z. Suo, G.M. Whitesides, Foldable Print. Circuit Boards Pap. Substr. Adv. Functl. Mater. 20 (2010) 28-35.

[4] J.A. Rogers, D.H. Kim, Stretchable elctronics: materials strategies and devices, Adv. Mater. 20 (2008) 4887-4892.

[5] A. Nathan, B.R. Chalamala, Special issue on flexible electronics technology, Part 1: systems and applications, Proc. leee. 93 (2005) (1235-1138).

[6] A. Nathan, A. Ahnood, M.T. Cole, S. Lee, Y. Suzuki, P. Hiralal, Flexible electronics: the next ubiquitous platform, Proc. IEEE 100 (2012) 1486-1517.

[7] W.M. Bruno, W.B. Bridges, Flexible dielectric waveguides with powder cores, IEEE Trans. Microw. Theory Tech. 36 (1988) 882-890.

[8] Z.M. Dang, H.Y. Wang, Y.H. Zhang, J.Q. Qi, Morphology and dielectric property of homogenous BaTiO3/PVDF nanocomposites prepared via the natural adsorption action of nanosized BaTiO3, Macromol. Rapid Commun. 26 (2005) 1185-1189.

[9] S. Shindo, I. Ohtomo, A coaxial type dielectric waveguide for $100 \mathrm{GHz}$ band. Tech. Rep. IECE Japan, MW 75105 (1975) 75-80.

[10] J. Obrzut, P.F. Goldsmith, Flexible circular waveguides at millimeter wavelengths from metallized Teflon tubing, IEEE Trans. Microw. Theory Tech. 38 (1990) 324-327. 
[11] M.T. Sebastian, H. Jantunen, Polymer-ceramic composites of 0-3 connectivity for circuits in electronics: a review, Int. J. Appl. Ceram. Technol. 7 (4) (2010) 415-434.

[12] D.K. Das -Gupta, C.J. Dias. Inorganic Ceramic / Polymer Ferroelectric Composite Electrets, October. 3, 1996, pp. 706-734.

[13] R. Popielarz, C.K. Chiang, R. Nozaki, J. Obrzut, Dielectric properties of polymer/ferroelectric ceramic composites from $100 \mathrm{~Hz}$ to $10 \mathrm{GHz}$, Macromolecules 34 (2001) 5910-5915.

[14] F. Xiang, H. Wang, X. Yao, Dielectric properties of SrTiO3/POE flexible composites for microwave applications, J. Eur. Ceram. Soc. 27 (2007) 3093-3097.

[15] D. Thomas, J. Chameswary, M.T. Sebastian, Mechanically flexible butyl rubber-SrTiO3 composites for microwave applications, Int. J. Appl. Ceram. Technol. 8(2011) 1099-1107.

[16] M.T., Sebastian, J., Chameswary, Poly(Isobutylene-co-Isoprene) Composite for Flexible Electronic Applications. Springer International Publishing Switzerland. D. Ponnamma et al. (eds.), Flexible and Stretchable Electronic Composites, Springer Series on Polymer and Composite Materials, 2016, pp 335-365

[17] G. Koulouridis, Y. Kiziltas, D.J. Zhou, Hansford, J.L. Volakis, Polymer-ceramic composites for microwave applications: fabrication and performance assessment, IEEE Trans. Microw. Theory Technol. 54 (2006) 4202-4208.

[18] S. Salaeh, N. Muensit, P. Bomlai, C. Nakason, Ceramic/natural rubber composites: influence types of rubber and ceramic materials on curing, mechanical, morphological, and dielectric properties, J. Mater. Sci. 46 (2011) 1723-1731.

[19] S.F. Wang, Y.R. Wang, K.C. Cheng, Y.P. Hsaio, Characteristics of polyimide/barium titanate composite films, Ceram. Intl. 35 (2009) 265-268.

[20] B. Agoudjil, L. Ibos, Y. Candau, J.C. Majeste, A comparative analysis of dielectric, rheological and thermophysical behaviour of Ethylene Vinyl Acetate/BaTiO3 composites, J. Phys. D: Appl. Phys. 41 (2008) 055407.

[21] P.L. Wise, I.M. Reaney, W.E. Lee, T.J. Price, D.M. Iddles, D.S. Cannell, Structuremicrowave property relations of Ca and Sr titanates, J. Eur. Ceram. Soc. 21 (2001)(2001) 2629.

[22] S. Rajesh, K.P. Murali, K.V. Rajani, R. Ratheesh, SrTiO3-Filled PTFE composite laminates for microwave substrate applications, Int. J. Appl. Ceram. Technol. 6 (2009) 553-561.

[23] V.S. Nisa, S. Rajesh, K.P. Murali, V. Priyadarsini, S.N. Potty, R. Ratheesh, Preparation, characterization and dielectric properties of temperature stable SrTiO3/PEEK composites for microwave substrate applications, Compos. Sci. Technol. 68 (2008) 106-112.

[24] H.-L. Chen, C.-L. Huang, Microwave dielectric properties and microstructures of Ca1-xNd2x-3TiO3Li1/2Nd1/2TiO3 ceramics, Jpn. J. Appl. Phys. 41 (2002) 5650-5653.

[25] M. Yoshida, N. Hara, T. Takada, A. Seki, Structure and properties of $\mathrm{Ca}(1-x N d 2 x / 3 T i O 3$, Jpn. J. Appl. Phys. 36 (1997) 6818-6823.

[26] W. Zhou, S. Qi, H.Z. Zhao, N.L. Liu, Thermally conductive silicone rubber reinforced with boron nitride particle, Polym. Compos. 28 (2007) 23-28. 
[27] L. Zhang, Z.Y. Cheng, Development of polymer-based 0-3 composites with high dielectric constant, J. Adv. Dielectr. 1 (2011) 389-406.

[28] C. Brosseau, P. Queffelec, P. Talbot, Microwave characterization of filled polymers, J. Appl. Phys. 89 (2001) 4532-4540.

[29] N. Jayasundere, B.V. Smith, Dielectric Constant for Binary Piezoelectric 0-3 Composites, J. Appl. Phys. 73 (1993) 2462-2466.

[30] R. Popielarz, C.K. Chiang, Polymer composites with dielectric constant comparable to that of barium titanate ceramics, Mater. Sci. Eng. B 139 (2007) 48-54.

[31] Z.M. Dang, Y.F. Yu, H.P. Xu, J. Bai, Study on microstructure and dielectric property of the BaTiO3/epoxy resin composites, Comp. Sci. Tech. 68 (2008) 171-177.

[32] J.C., Maxwell Garnett, Colours in Metal Glasses and in Metallic Films. Phil. Trans. Royal Society of London. Series A. Containing Papers of a Mathematical or Physical Character, 203, 1904, pp. 385-420.

[33] K. Lichtenecker, K. Rother, Die herleitung des logarithm mischenmischungsgesetzesaus allegemeinen prinzipien der stationaren stromung, Phys. Z. 32 (1931) 255-260.

[34] Y. Rao, J. Qu, T.C. Marinis, C.P. Wong, A precise numerical prediction of effective dielectric constant for polymer-ceramic composite based on effective-medium theory, IEEE Trans. Compon. Packag. Technol. 23 (2000) 680-683.

[35] K. Wakino, T. Okada, N. Yoshida, K. Tomono, A new equation for predicting the dielectric-constant of a mixture, J. Am. Ceram. Soc. 76 (1993) 2588.

[36] P.S. Anjana, M.T. Sebastian, M.N. Suma, P. Mohanan, Low dielectric loss PTFE/CeO2 ceramic composites for microwave substrate applications, Int. J. Appl. Ceram. Technol. 5 (2008) 325.

[37] I. Vrejoiu, J.D. Pedarnig, M. Dinescu, S. Bauer-Gogonea, D. Bäuerle, Flexible ceramic-polymer composite films with temperature-insensitive and tunable dielectric permittivity, Appl. Phys. A. 74 (2002) 407-409.

[38] S.H. Xie, B.K. Zhu, X.Z. Wei, Z.K. Xu, Y.Y. Xu, Polyimide/ BaTiO3 composites with controllable dielectric properties, Compos. Part A 36 (2005) 1152-1157.

[39] M.A. Berger, R.L. Mc Cullough, Characterization and analysis of the electrical properties of a metalfilled polymer, Compos. Sci. Technol. 22 (1985) 81-106.

[40] S. Kamba, M. Savinov, F. Laufek, O. Tkac, C. Kadlec, S. Veljko, E.J. John, G. Subodh, M.T. Sebastian, M. Klementova, V. Bovtun, J. Pokorny, V. Goian, J. Petzelt, Chem. Mater. 21 (2009) 811-819.

[41] L. Holliday, Robinson, The thermal expansion of composites based on polymers, J. Mater. Sci. 8 (1973) 301-311.

[42] G.W. Lee, M. Park, J. Kim, J.I. Lee, H.G. Yoon, Enhanced thermal conductivity of polymer composites filled with hybrid fille, Compos. Part A 37 (2006) 727-734.

[43] G. Subodh, M.V. Manjusha, J. Philip, M.T. Sebastian, Thermal properties of polytetrafluoroethylene/Sr2Ce2Ti5O16 polymer/ceramic composite, J. Appl. Polym. Sci. 108 (2008) 17161721. 
[44] T.S. Laverghetta, Microwave materials and fabrication techniques 3rd Edition, IEEE Aerosp. Electron. Syst. Mag. 17 (2002).

\section{Tables and Figures}

Table 1. Dielectric and physical properties of ceramic fillers.

\begin{tabular}{|l|l|l|l|l|l|l|}
\hline Material & $\begin{array}{l}\text { Density } \\
\left(\mathbf{g} / \mathbf{c m}^{3}\right)\end{array}$ & $\begin{array}{l}\text { Avg. Particle } \\
\text { size }(\boldsymbol{\mu m})\end{array}$ & $\begin{array}{l}\text { Relative } \\
\text { permittivity } \varepsilon_{\mathbf{r}}\end{array}$ & $\begin{array}{l}\text { Dielectric loss } \\
\text { tan } \delta\end{array}$ & $\begin{array}{l}\text { Coefficient of } \\
\text { thermal } \\
\text { expansion } \\
\left(\mathbf{p p m} /{ }^{\circ} \mathbf{C}\right)\end{array}$ & $\begin{array}{l}\text { Thermal } \\
\text { conductivity } \\
\left(\mathbf{W m}^{-1} \mathbf{K}^{-1}\right)\end{array}$ \\
\hline $\mathrm{BaTiO}_{3}$ & 6.01 & 0.90 & $1250($ at $1 \mathrm{MHz})$ & $10^{-2}($ at $1 \mathrm{MHz})$ & 5.4 & 2.6 \\
\hline $\mathrm{SrTiO}_{3}$ & 5.12 & 0.80 & $290.0($ at $1 \mathrm{GHz})$ & $10^{-3}($ at $1 \mathrm{GHz})$ & 9.4 & 12.0 \\
\hline $\mathrm{Ca}(1-x) \mathrm{Nd}_{(2 \times / 3)} \mathrm{TiO}_{3}$ & 4.57 & 0.90 & $98.0($ at $7 \mathrm{GHz})$ & $10^{-3}($ at $7 \mathrm{GHz})$ & 10.3 & 2.4 \\
\hline
\end{tabular}

Table 2. The formulations of silicone rubber composites (Ingredients in $\mathrm{phr}^{\mathrm{a}}$ ).

\begin{tabular}{|c|c|c|c|c|}
\hline Composite material & $\begin{array}{l}\text { Sample } \\
\text { designation }\end{array}$ & $\begin{array}{l}\text { Silicone rubber } \\
\text { in } \text { phr }^{\mathrm{a}}\end{array}$ & $\begin{array}{l}\text { Dicumyl peroxide } \\
\text { (DCP) in } \mathrm{phr}^{\mathrm{a}}\end{array}$ & Filler in $p r^{a}[]^{b}$ \\
\hline \multirow{5}{*}{$\begin{array}{l}\text { Silicone rubber Batio3 (SR- } \\
\text { BT) }\end{array}$} & $\mathrm{SRBT}_{0}$ & 100 & 2 & $0[0]$ \\
\hline & $\mathrm{SRBT}_{1}$ & 100 & 2 & $10[0.04]$ \\
\hline & $\mathrm{SRBT}_{2}$ & 100 & 2 & $50[0.09]$ \\
\hline & $\mathrm{SRBT}_{3}$ & 100 & 2 & $100[0.16]$ \\
\hline & $\mathrm{SRBT}_{4}$ & 100 & 2 & $200[0.28)$ \\
\hline \multirow{5}{*}{$\begin{array}{l}\text { Silicone rubber SrTiO3 (SR- } \\
\text { ST) }\end{array}$} & $\mathrm{SRST}_{0}$ & 100 & 2 & $0[0]$ \\
\hline & $\mathrm{SRST}_{1}$ & 100 & 2 & $10[0.02]$ \\
\hline & $\mathrm{SRST}_{2}$ & 100 & 2 & $50[0.09]$ \\
\hline & $\mathrm{SRST}_{3}$ & 100 & 2 & $100[0.18]$ \\
\hline & $\overline{\mathrm{SRST}_{4}}$ & 100 & 2 & $200[0.30]$ \\
\hline \multirow{5}{*}{$\begin{array}{l}\text { Silicone rubber } \mathrm{Ca}(1- \\
\text { x) Nd(2x/3)Tio3 (SRCNT) }\end{array}$} & $\mathrm{SRCT}_{0}$ & 100 & 2 & $0[0]$ \\
\hline & $\mathrm{SRCT}_{1}$ & 100 & 2 & $10[0.04]$ \\
\hline & $\mathrm{SRCT}_{2}$ & 100 & 2 & $50[0.16]$ \\
\hline & $\mathrm{SRCT}_{3}$ & 100 & 2 & $100[0.27]$ \\
\hline & $\mathrm{SRCT}_{4}$ & 100 & 2 & $200[0.43]$ \\
\hline
\end{tabular}

${ }^{\mathrm{a}}$ Parts per hundred of rubber.

${ }^{b}$ Volume fractions of filler given in parenthesis. 
Fig. 1. XRD patterns of (a) BT (b) ST and (c) CNT.

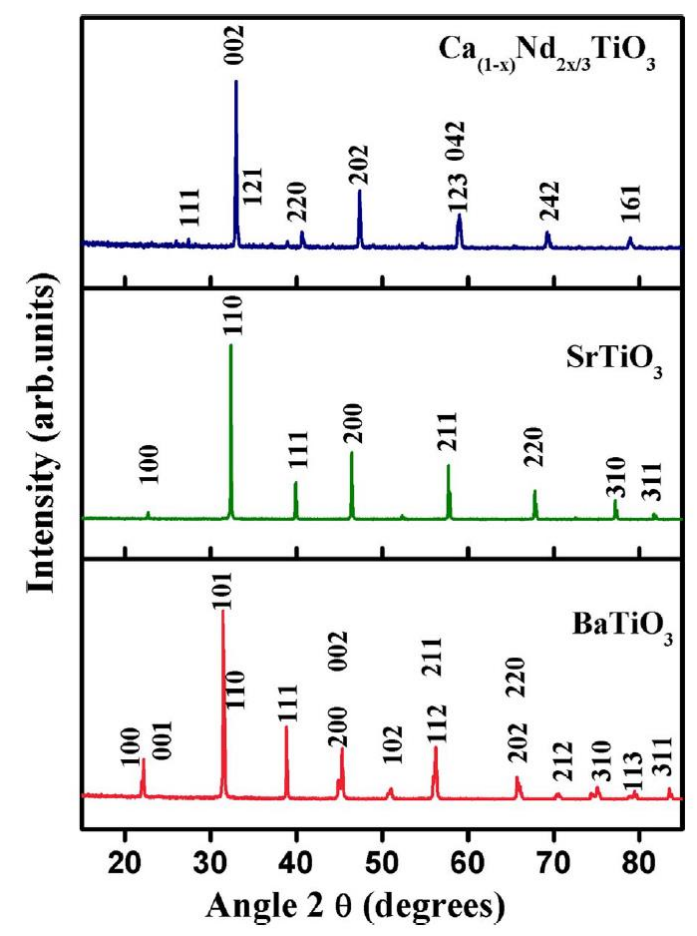

Fig. 2. SEM images of $\left(A_{1}\right) B T\left(B_{1}\right) S T\left(C_{1}\right) C T$ ceramics, $\left(A_{2}\right),\left(B_{2}\right)$ and $\left(C_{2}\right)$ fractograms of low filler loaded composites and $\left(A_{3}\right),\left(B_{3}\right)$ and $\left(C_{3}\right)$ fractograms of higher filler loaded SRBT, SRST and SRCT composites respectively.
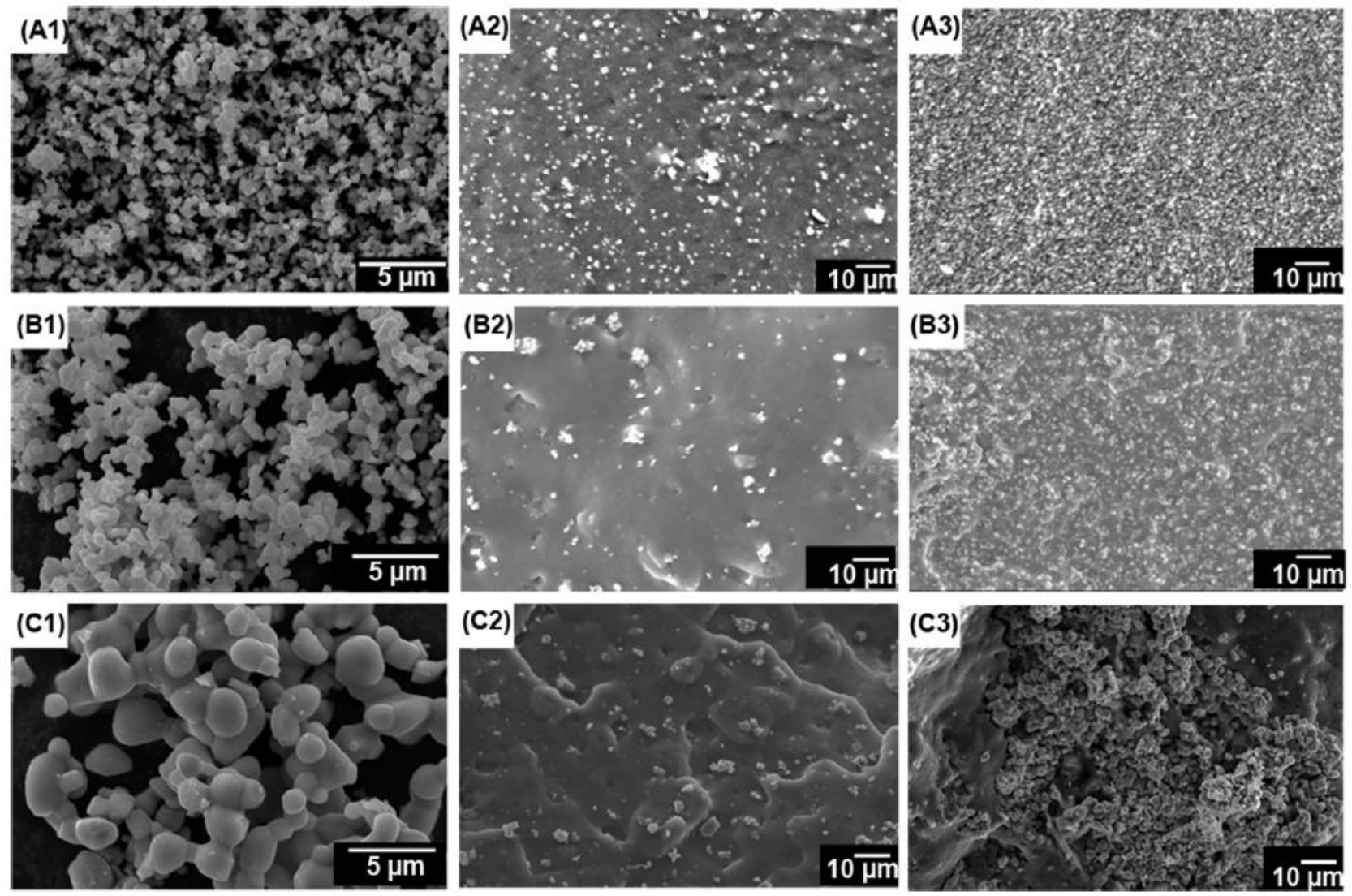
Fig. 3. Stress-strain curves of SRBT, SRST and SRCT composites.
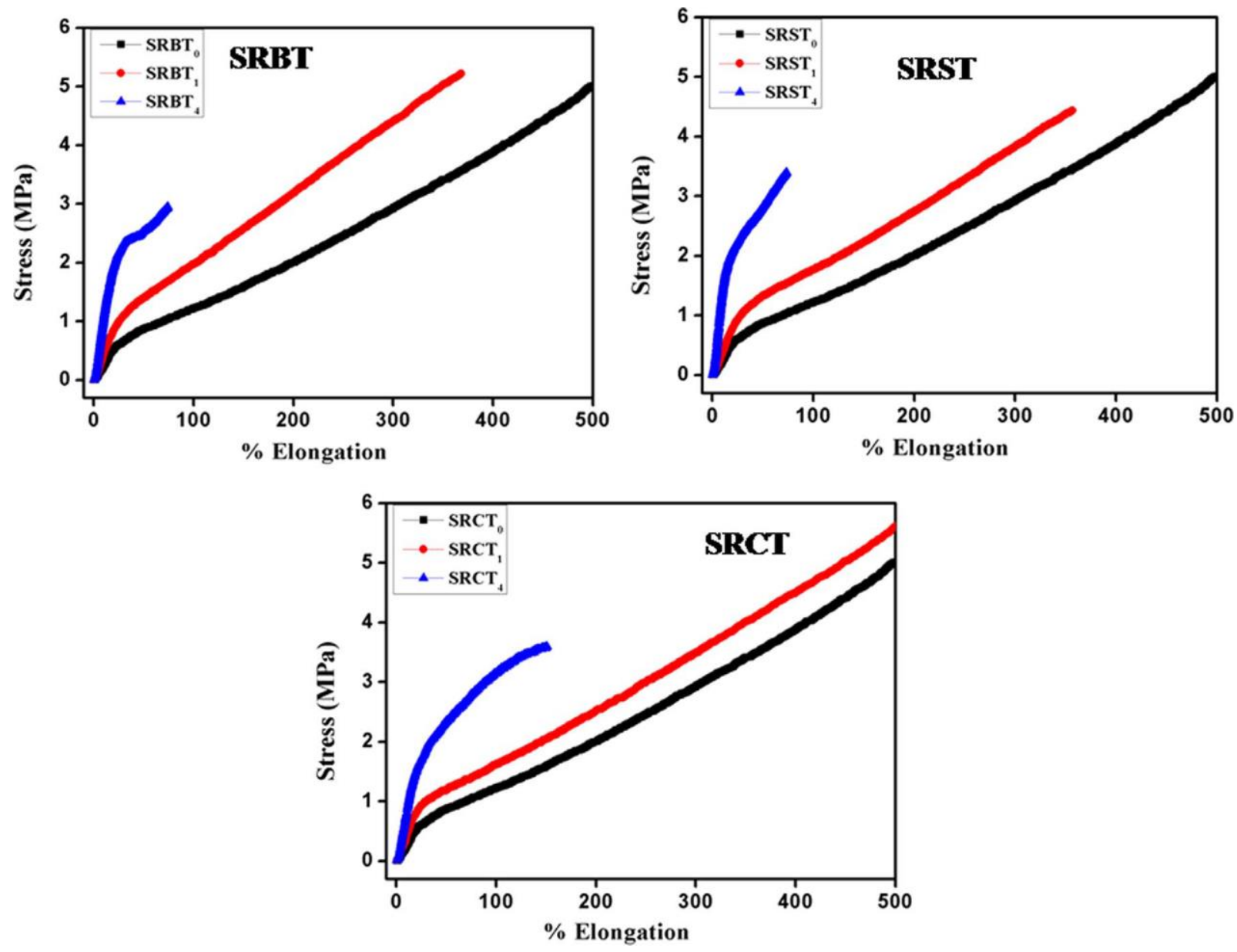

Fig. 4. Variations of dielectric properties of SR-BT, SR-ST and SR-CNT composites at (a)1 MHz, (b) $5 \mathrm{GHz}$ and (c) $15 \mathrm{GHz}$.
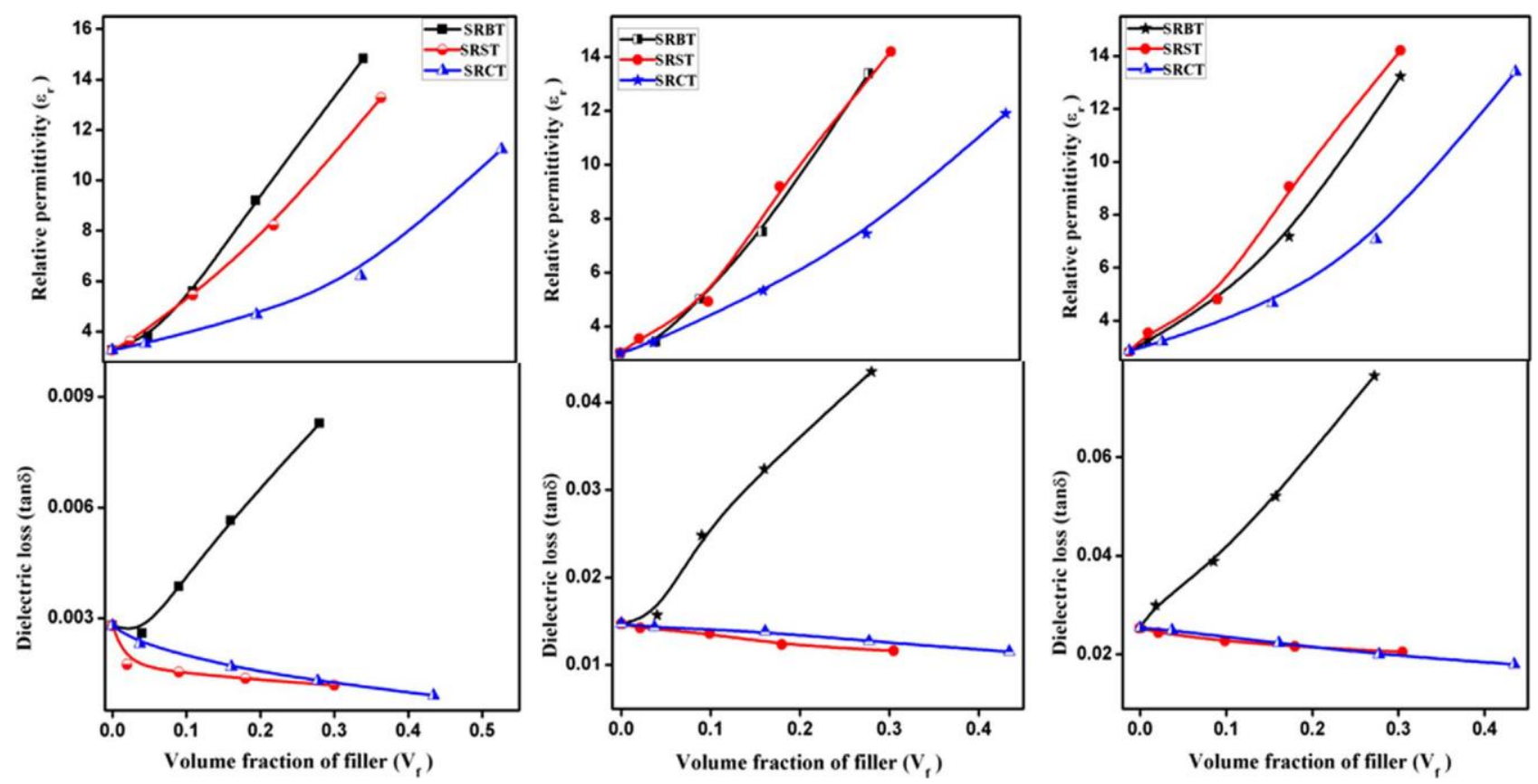
Fig. 5. Comparison of experimental and theoretical relative permittivity of (a) SR-BT (b) SR-ST and (c) SRCNT composites at $5 \mathrm{GHz}$.
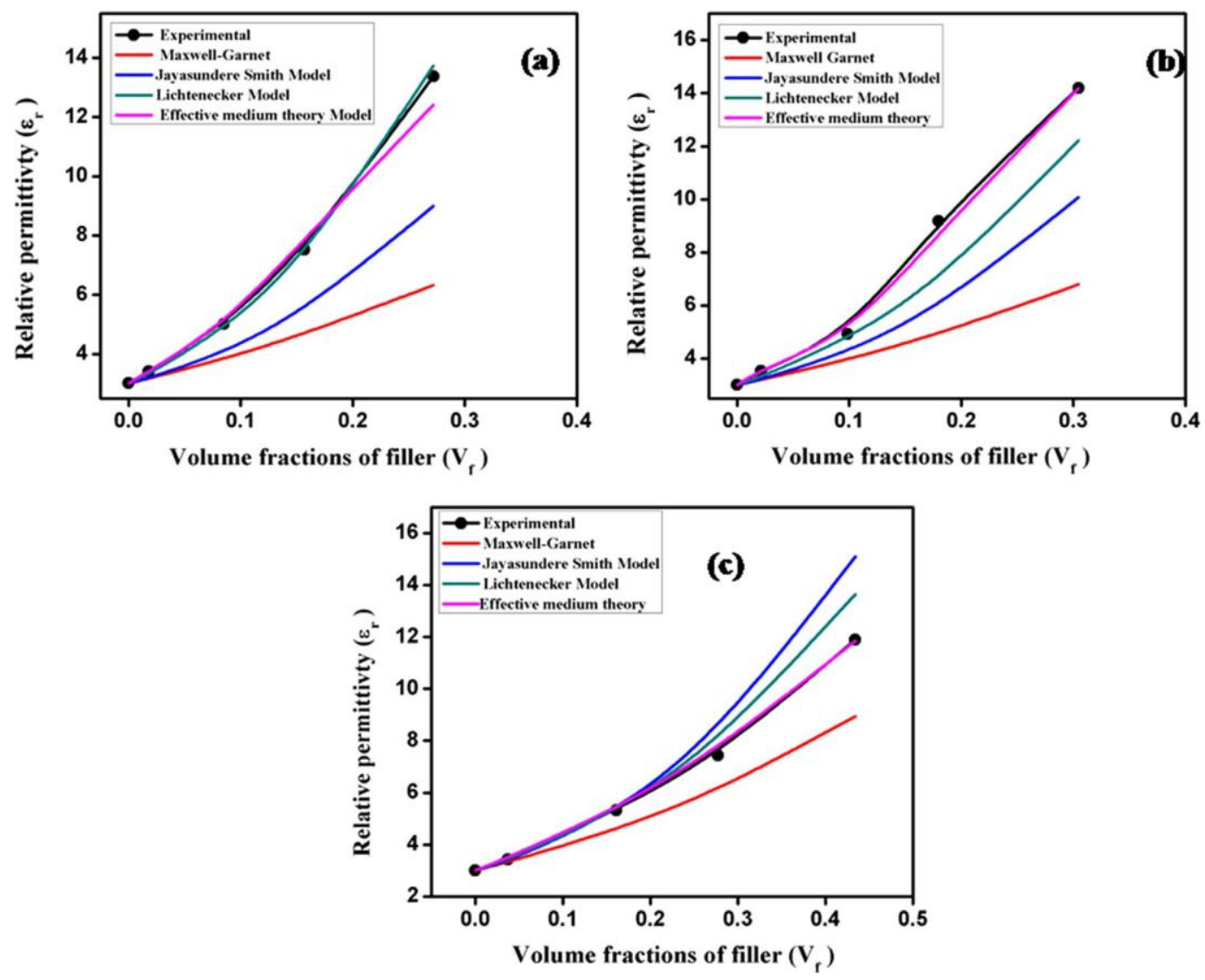
Fig. 6. Variation of dielectric properties of (a) SR-BT (b) SR-ST and (c) SR-CNT composites with bending.

SRBT

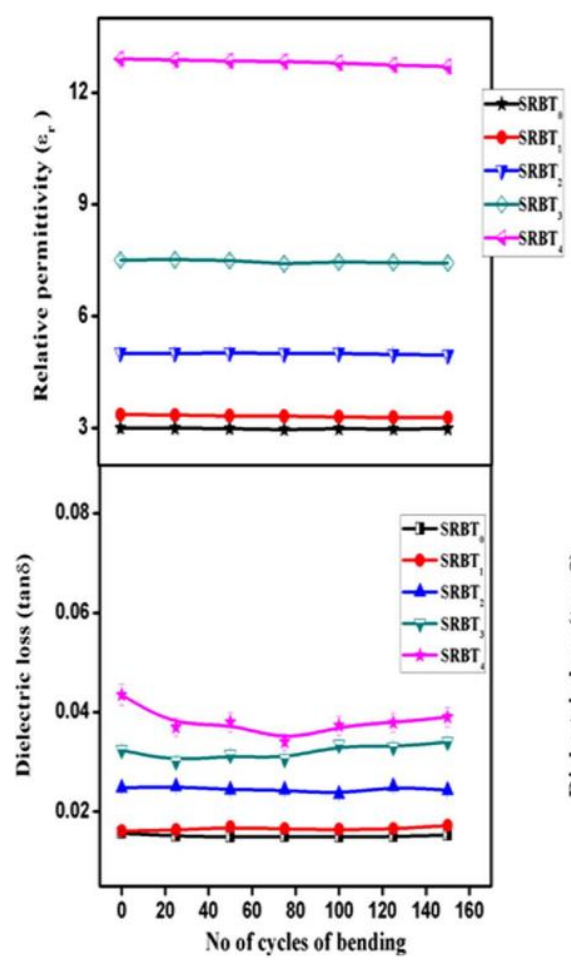

SRST

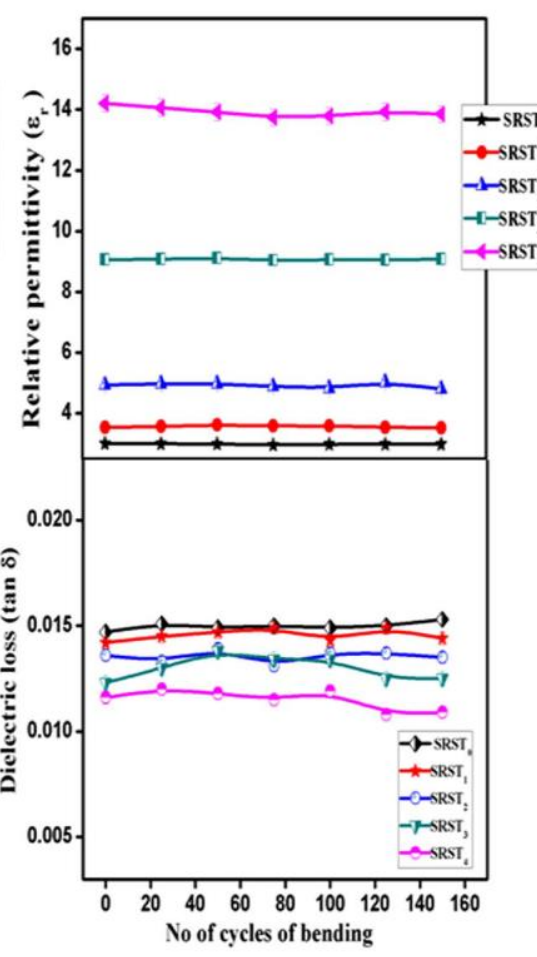

SRCT

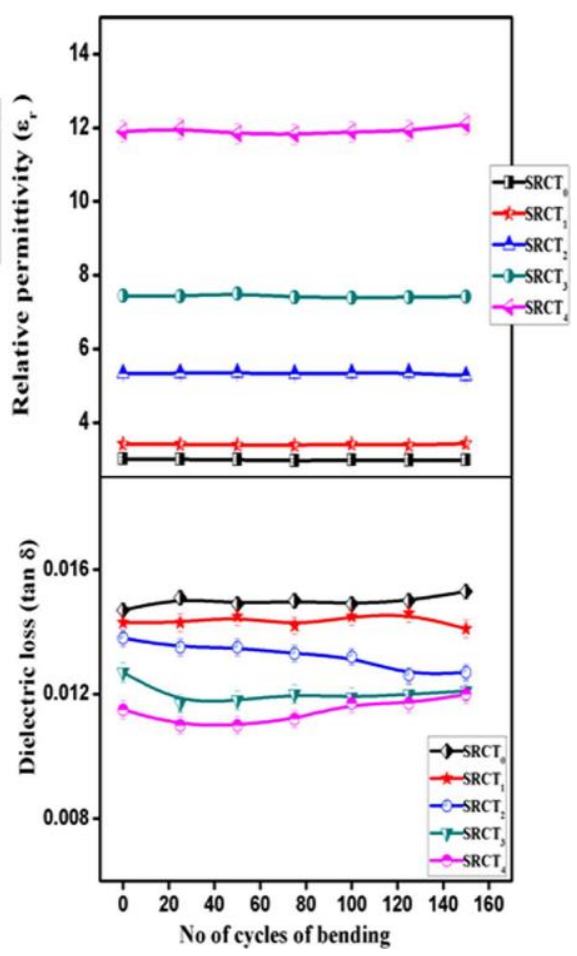

Fig. 7. The variation of relative permittivity of (a) SR-BT (b) SR-ST and (c) SR-CNT composite
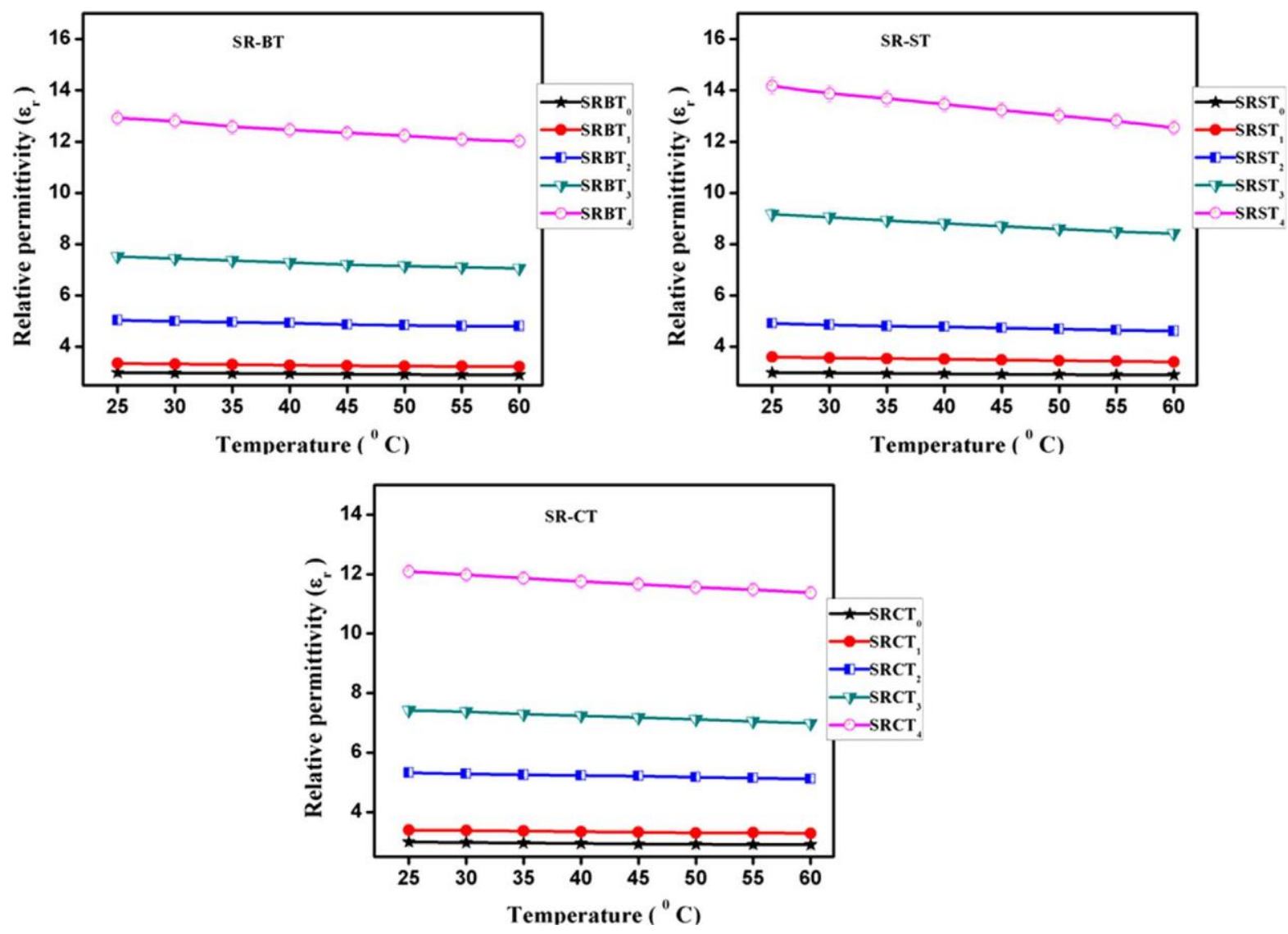
Fig. 8. Variation of coefficient of thermal expansion of (a) SR-BT, (b) SR-ST and (c) SRCNT composites with ceramic loading.

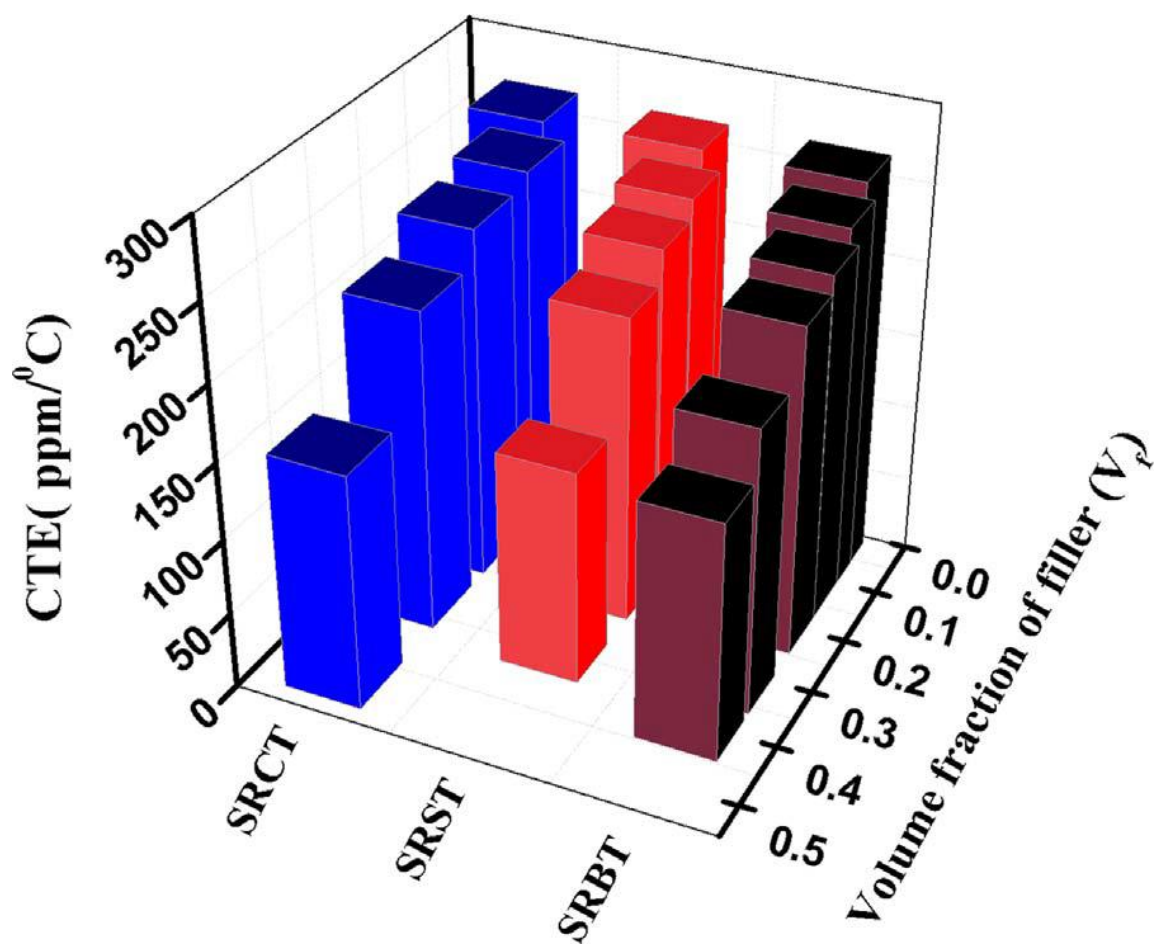

Fig. 9. Variation of thermal conductivity, specific heat capacity and thermal diffusivity of (a) SR-BT (b) SR-ST and (c) SR-CNT composite with filler volume fraction
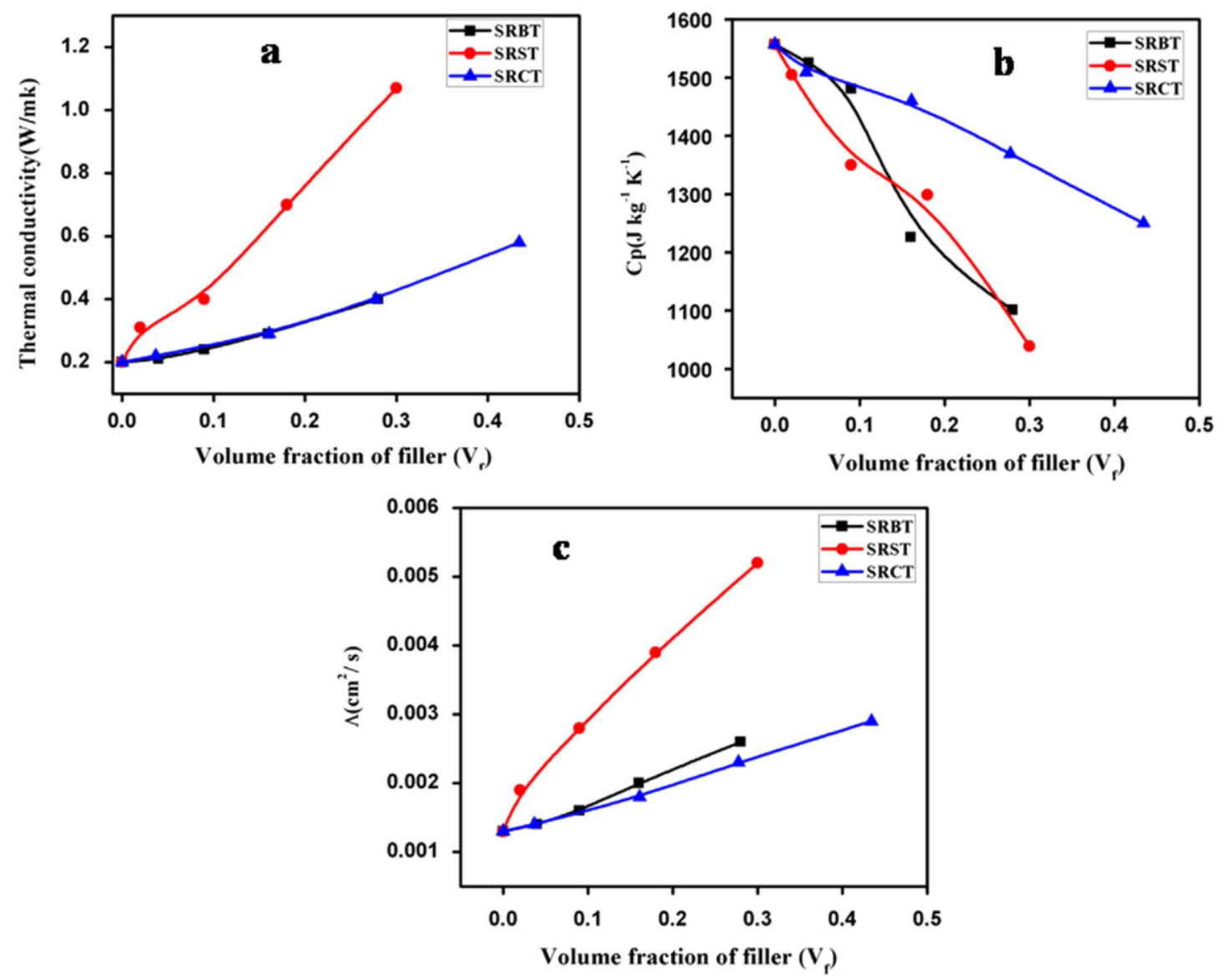
Fig. 10. Variation of water absorption of (a) SR-BT (b) SR-ST (c) SR-CNT composites with filler loading.

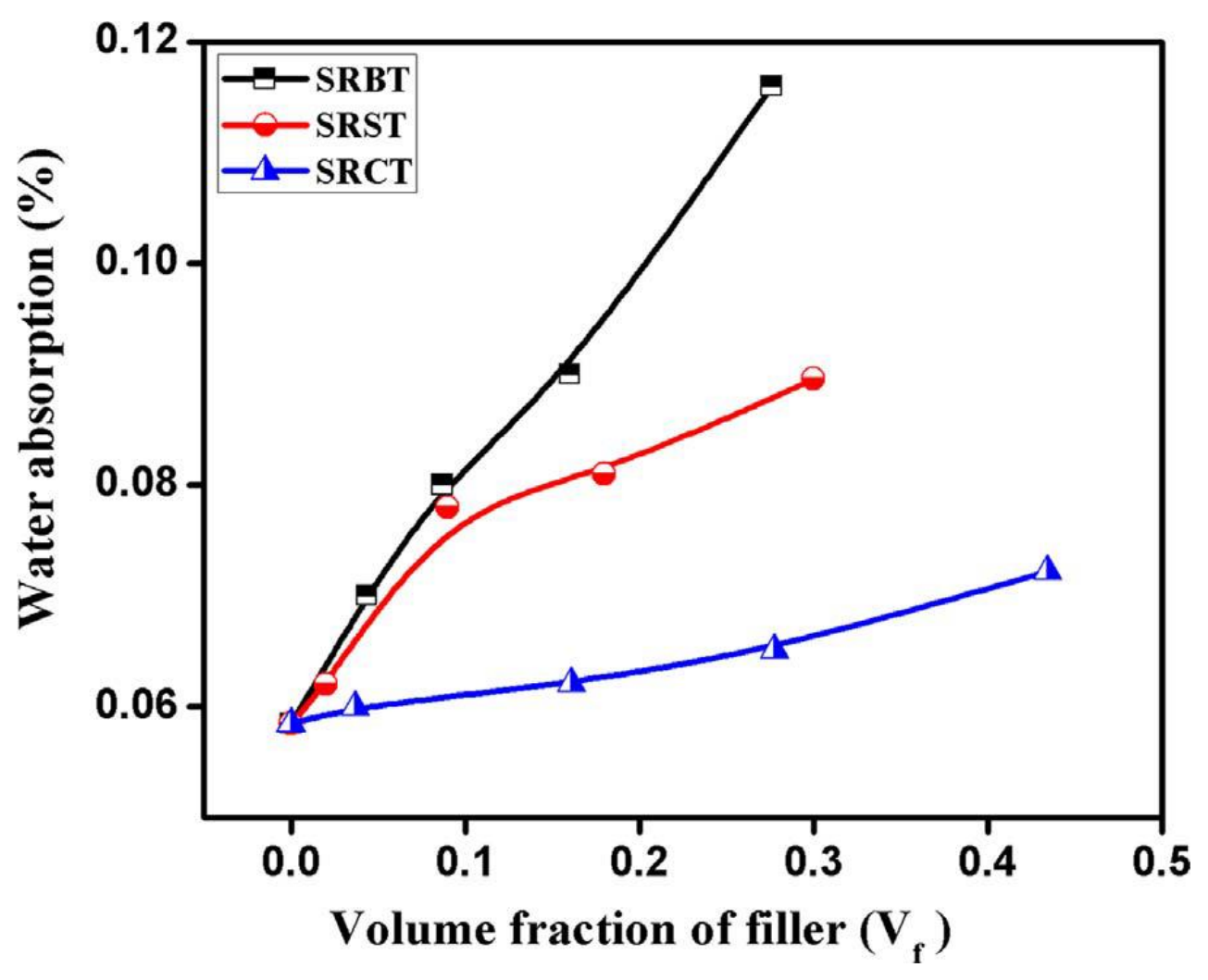

